\title{
Tightness of Semidefinite Programming Relaxation to Robust Transmit Beamforming with SINR Constraints
}

\author{
Yanjun Wang and Ruizhi Shi \\ Department of Applied Mathematics, Shanghai University of Finance \& Economics, Shanghai 200433, China \\ Correspondence should be addressed to Yanjun Wang; wangyj@mail.shufe.edu.cn
}

Received 21 June 2013; Revised 20 October 2013; Accepted 23 October 2013

Academic Editor: John Gunnar Carlsson

Copyright ( 2013 Y. Wang and R. Shi. This is an open access article distributed under the Creative Commons Attribution License, which permits unrestricted use, distribution, and reproduction in any medium, provided the original work is properly cited.

\begin{abstract}
This paper considers a multiuser transmit beamforming problem under uncertain channel state information (CSI) subject to SINR constraints in a downlink multiuser MISO system. A robust transmit beamforming formulation is proposed. This robust formulation is to minimize the transmission power subject to worst-case signal-to-interference-plus-noise ratio (SINR) constraints on the receivers. The challenging problem is that the worst-case SINR constraints correspond to an infinite number of nonconvex quadratic constraints. In this paper, a natural semidifinite programming (SDP) relaxation problem is proposed to solve the robust beamforming problem. The main contribution of this paper is to establish the tightness of the SDP relaxation problem under proper assumption, which means that the SDP relaxation problem definitely yields rank-one solutions under the assumption. Then the SDP relaxation problem provides globally optimum solutions of the primal robust transmit beamforming problem under proper assumption and norm-constrained CSI errors. Simulation results show the correctness of the proposed theoretical results and also provide a counterexample whose solutions are not rank one. The existence of counterexample shows that the guess that the solutions of the SDP relaxation problem must be rank one is wrong, except that some assumptions (such as the one proposed in this paper) hold.
\end{abstract}

\section{Introduction}

Multiple antenna transmission has been a promising approach for obtaining significant throughput gains in wireless broadcast channels [1]. This paper will focus on a standard unicast antenna receivers using transmit beamforming [2]. A popular measure of quality of service in a multiuser communication system is the signal-to-interference-plusnoise ratio (SINR). However, in the transmit beamforming designs, if SINR is used as the receivers' quality of service measure, the problem will be challenging because they often involve nonconvex optimization problems, which are known to be difficult in general. Recent developments have shown that the unicast transmit beamforming problem can actually be solved efficiently through proper problem reformulations and the convex optimization theory [2-4].

The efficacy of unicast transmit beamforming designs with SINR constraints relies on the channel state information (CSI) of the receivers. In wireless systems, CSI is either estimated via uplink training or acquired through receivers' feedback. Hence, it is inevitable to have CSI errors in practice due to finite-length training data or due to limited quantization feedback bandwidth. In these cases, the conventional beamforming designs may no longer guarantee the desired quality of service (QoS) requirements, and therefore the SINR outage may occur. Then, the transmit beamforming designs that take into consideration the CSI errors have been a research subject drawing great interests in recent years.

There are two classes of transmit beamforming designs that consider the CSI errors: the stochastic design $[5,6]$ and robust design (worst case) [7-12]. In the stochastic design, the CSI errors are modeled as random vectors, and the design formulation where the transmission power is minimized is subject to probability SINR constraints. The probabilistic SINR constraints guarantee that the beamforming design provides each receiver with a preset, often high, probability that the desired SINR specification is satisfied (i.e., a high SINR satisfaction probability). The associated design problem 
is difficult because the probabilistic SINR constraints in general do not possess any tractable form. In the robust designs, CSI errors are assumed to be bounded within a predetermined small error set. Robust transmit beamforming design aims to find a most power-efficient beamforming design that guarantees the receiver $k$ a 100\% SINR satisfaction probability for all channels satisfying this bounded error model. It is seen that this formulation is a conservative approach. In other words, the robust beamforming formulation that minimizes the transmission power is subject to worst-case SINR constraints on all the receivers. Robust formulation is a challenging task because the problem has infinite number of deterministic SINR constraints and is nonconvex. To overcome the computational difficulties associated with the robust downlink beamforming, researchers have proposed various optimization methods to approximate the problem by restricting the nonconvex feasible region to a small convex set [11]. However, due to convex restriction, these methods cannot necessarily reach the global optimal solution although they do guarantee the satisfaction of the SINR constraints.

This paper adopts a natural SDP relaxation problem denoted by (SDR) to solve the robust beamforming problem. It is known that SDP relaxation has been widely used in the context of transmit beamforming $[13,14]$. Also the (SDR) of the robust beamforming problem has also been derived and empirically tested in $[10,12,15,16]$. Some authors found an interesting fact that the (SDR) often yields rank-one solutions when the uncertainty regions are balls or ellipsoidal (as far as the problem is feasible). However, in [17], the authors present three counterexamples under ellipsoidal uncertainty regions whose solutions are not rank one accordingly. Hence, a key issue is whether the problem (SDR) can exactly solve the original robust problem under proper assumption. Although the SDP relaxation problem for nonrobust unicast transmit beamforming has been shown to be an exact solver [2, 3], it has not been known whether the same desirable result holds for robust design. In [17], authors have shown that the solution of the (SDR) is always rank one if the error bound is sufficiently small. In [18], the authors propose sufficient conditions which can assure the tightness of the SDP relaxation. The conditions in [18] are not expressed in terms of the data of the problems, which is an assumption for all the feasible solutions. Inspired by the previous work, in this paper, we try to get the insight into what is happening at the optimal solution and the optimal error vector $\delta_{k}^{*}$ and propose a new assumption condition. This condition is that at the optimal solution there exists a unique error vector $\delta_{k}^{*}$ which satisfies some necessary conditions. The main goal of this paper is to show under this condition that the (SDR) problem is tight (i.e., giving a rank-one solution) when the uncertainty regions are balls. In particular, we will prove that under this condition the solution of (SDR) must be rank one; that is, the (SDR) provides globally optimum solutions of the primal robust transmit beamforming problem. Also in the numerical simulation, a counterexample is provided whose solutions are not rank one. Hence, we can conclude that the guess that the solutions of the (SDR) must be rank one is wrong, except that some assumptions (such as the one proposed in this paper) hold.
The structure of this paper is organized as follows. In Section 2, the signal model and the robust SINR constrained transmit beamforming problem are presented. Section 3 presents the semidefinite relaxation problem (SDR) of the primal robust model. Some properties for (SDR) are explored in this section, especially the properties at the optimal solution. Section 4 shows that the problem (SDR) will yield rankone solution under proper assumption and then provides globally optimal solutions of the primal problem. Simulation results are provided in Section 5. And Section 6 is the conclusion.

Notations. The notation $H^{n \times n}$ stands for the set of $n$ by $n$ complex Hermitian matrices, and $C^{n}$ stands for the sets of $n$-dimensional complex vectors. The superscript " $H$ " represents the Hermitian transpose; and $A \geq 0(>0)$ means that matrix $A$ is positive semidefinite (positive definite). $\operatorname{Tr}(A)$ and $\operatorname{rank}(A)$ denote the trace and rank of matrix $A$, respectively. $\|\cdot\|$ represents the vector Euclidean norm.

\section{Signal Model and Problem Formulation}

2.1. Signal Model. We consider a K-user downlink MISO system where the base station (BS) is equipped with $M$ antennas. The BS wants to transmit $K$-independent data streams $s_{1}, s_{2}, \ldots, s_{k}$ to $K$ users and each $s_{K}$ is complex Gaussian with unit variance. The BS uses linear beamforming vector $g_{k} \in C^{M}, k=1, \ldots, K$, to send the stream $s_{K}$. The received signal at receiver $k$ is

$$
\widehat{s}_{k}=h_{k}^{H} \sum_{i=1}^{K} s_{i} g_{i}+\omega_{k}, \quad k=1, \ldots, K
$$

where $h_{k}$ denotes the channel vector from BS to the $k$ th user. $\left\{\omega_{k}\right\}$ are zero-mean independent white complex Gaussian noises, each with a variance of $\sigma_{k}^{2}>0$. Therefore, the SINR of the $k$ th user is

$$
\operatorname{SINR}_{k}=\frac{\left|h_{k}^{H} g_{k}\right|^{2}}{\sum_{i \neq k}\left|h_{k}^{H} g_{i}\right|^{2}+\sigma_{k}^{2}} .
$$

Since the SINR is directly related to system performance such as the bit error rate, it is commonly used as the receivers' quality-of-service measure. The goal of the transmitter is to design the beamforming vectors $\left\{g_{i}\right\}_{i=1}^{K}$ such that each of the receivers can meet a desired SINR requirement as

$$
\operatorname{SINR}_{k} \geq \gamma_{k}, \quad k=1, \ldots, K
$$

where $\gamma_{k}>0$ is the target SINR value for the receiver $k$.

2.2. Nonrobust Transmit Beamforming Problem. The transmitter requires the receivers' CSI, that is, $\left\{h_{i}\right\}_{i=1}^{K}$. Suppose that the transmitter perfectly knows $\left\{h_{i}\right\}_{i=1}^{K}$. Then, the optimum 
beamforming vectors can be obtained by solving the following optimization problem:

$$
\begin{array}{ll}
\min _{\left\{g_{k}\right\}_{k=1}^{K} \in C^{M}} & \sum_{k=1}^{K}\left\|g_{k}\right\|^{2} \\
\text { s.t. } & \frac{\left|h_{k}^{H} g_{k}\right|^{2}}{\sum_{i \neq k}\left|h_{k}^{H} g_{i}\right|^{2}+\sigma_{k}^{2}} \geq \gamma_{k}, \\
& k=1, \ldots, K .
\end{array}
$$

The problem $(\mathrm{P})$ can be rewritten into

$$
\begin{array}{ll}
\min _{\left\{G_{k}\right\}_{k=1}^{K}} & \sum_{k=1}^{K} \operatorname{Tr}\left(G_{k}\right) \\
\text { s.t. } & \frac{h_{k}^{H} G_{k} h_{k}}{\sum_{i \neq k} h_{k}^{H} G_{i} h_{k}+\sigma_{k}^{2}} \geq \gamma_{k}, \\
& G_{k}=g_{k} g_{k}^{H}, \quad k=1, \ldots, K .
\end{array}
$$

By replacing these nonconvex constraints $G_{k}=g_{k} g_{k}^{H}$ by the following constraint: $G_{k} \geq 0$, then we can obtain the following relaxation programming:

$$
\begin{array}{ll}
\min _{\left\{G_{k}\right\}_{k=1}^{K}} & \sum_{k=1}^{K} \operatorname{Tr}\left(G_{k}\right) \\
\text { s.t. } & \frac{h_{k}^{H} G_{k} h_{k}}{\sum_{i \neq k} h_{k}^{H} G_{i} h_{k}+\sigma_{k}^{2}} \geq \gamma_{k}, \quad k=1, \ldots, K, \\
& G_{k} \geq 0, \quad k=1, \ldots, K .
\end{array}
$$

The problem (PR) is convex, which means that any local minima must be a global minima and the set of optimal solutions is convex (typically a single point). Obviously, the problem (PR) is also a SDP problem. A number of so-called interior point methods or central-path methods have appeared that can solve SDP problems efficiently. Also, several program packages are freely available, for example, CVX, SeDuMi, and YALMIP. Usually, the optimum of (PR) will provide a lower bound on the optimum of $(\mathrm{P})$. However, the specific structure of (PR) enables us to have the following remarkable result $[2,3]$. The problem $(\mathrm{PR})$ does always have at least one optimal solution where all $G_{k}(k=1, \ldots, K)$ are rank one. This result shows that (PR) is not only a semidefinite relaxation, but also an equivalent reformulation of the problem $(\mathrm{P})[2,3]$.

2.3. Robust Transmit Beamforming Problem. In wireless systems, CSI at the transmitter is either estimated via uplink training or acquired through receivers' feedback. Hence, it is inevitable to have CSI errors in practice due to the finite-length training data or due to the limited quantization feedbacks. Let $\widetilde{h}_{k}(k=1, \ldots, K)$ denote the obtained channel estimates at the transmitter. The channel vector $h_{k}$ lies within a ball with radius $\epsilon_{k}$ around the estimated channel vector $\widetilde{h}_{k}$; that is,

$$
h_{k} \in U_{k}=\left\{\tilde{h}_{k}+\delta_{k} \mid\left\|\delta_{k}\right\| \leq \epsilon_{k}\right\}
$$

for all $k=1, \ldots, K$, where $\delta_{k} \in C^{M}$ is the channel estimation error whose norm is assumed to be bounded by $\epsilon_{k}$. With the imperfect CSI, the standard transmit beamforming designs in (P) may no longer guarantee the desired SINR specifications in (3), and therefore the SINR outage may occur.

When the optimization problem is in the face of data uncertainty in the constraints, robust optimization is one of the powerful deterministic approaches. Within the framework of robust optimization method, the robust counterpart is presented as follows:

$$
\begin{array}{ll}
\min _{\left\{g_{k}\right\}_{k=1}^{K} \in C^{M}} & \sum_{k=1}^{K}\left\|g_{k}\right\|^{2} \\
\text { s.t. } & \frac{\left|h_{k}^{H} g_{k}\right|^{2}}{\sum_{i \neq k}\left|h_{k}^{H} g_{i}\right|^{2}+\sigma_{k}^{2}} \geq \gamma_{k}, \quad \forall h_{k} \in U_{k}, \\
& \quad k=1, \ldots, K .
\end{array}
$$

The problem (RP) aims to find a most power-efficient beamforming design that guarantees the receiver $k$ a $100 \%$ SINR satisfaction probability for all uncertain cases of $\left\{h_{k}\right\}$. It is seen that this formulation is a conservative approach. Solving problem (RP) is a challenging task because the problem has infinite number of deterministic SINR constraints and is nonconvex. Similar to the method in deterministic case, the problem (RP) can be rewritten into the following form:

$$
\begin{array}{ll}
\min _{\left\{G_{k}\right\}_{k=1}^{K}} & \sum_{k=1}^{K} \operatorname{Tr}\left(G_{k}\right) \\
\text { s.t. } & \frac{h_{k}^{H} G_{k} h_{k}}{\sum_{i \neq k} h_{k}^{H} G_{i} h_{k}+\sigma_{k}^{2}} \geq \gamma_{k}, \quad \forall h_{k} \in U_{k}, \\
& G_{k}=g_{k} g_{k}^{H}, \quad k=1, \ldots, K .
\end{array}
$$

By replacing these nonconvex constraints $G_{k}=g_{k} g_{k}^{H}$ by the following constraint: $G_{k} \geq 0$, then we can obtain the following convex relaxation programming:

$$
\begin{array}{ll}
\min _{\left\{G_{k}\right\}_{k=1}^{K}} & \sum_{k=1}^{K} \operatorname{Tr}\left(G_{k}\right) \\
\text { s.t. } & \frac{h_{k}^{H} G_{k} h_{k}}{\sum_{i \neq k} h_{k}^{H} G_{i} h_{k}+\sigma_{k}^{2}} \geq \gamma_{k}, \quad \forall h_{k} \in U_{k} \\
& G_{k} \geq 0, \quad k=1, \ldots, K .
\end{array}
$$

Since the problem (RPR) aims to find the most powerefficient beamforming design that guarantees the receiver $k$ a $100 \%$ SINR satisfaction probability for all uncertain cases of $\left\{h_{k}\right\}$, then the question whether the (RPR) is feasible is highly related to the bound $\epsilon_{k}$ which is bounding the channel estimation error $\delta_{k}$. The following lemma provides a necessary condition for the feasibility of the problem (RPR). 
Lemma 1. If the (RPR) problem is feasible, then it must hold that $\epsilon_{k}<\left\|\widetilde{h}_{k}\right\|^{2}$ for all $k=1, \ldots, K$.

Proof. Suppose that there exists an index $k_{0}$ such that $\epsilon_{k_{0}} \geq$ $\left\|\widetilde{h}_{k_{0}}\right\|^{2}$. Since (RPR) is feasible, then for $k=k_{0}$, it must hold that

$$
\frac{h_{k_{0}}^{H} G_{k_{0}} h_{k_{0}}}{\sum_{i \neq k_{0}} h_{k_{0}}^{H} G_{i} h_{k_{0}}+\sigma_{k_{0}}^{2}} \geq \gamma_{k_{0}}, \quad \forall h_{k_{0}} \in U_{k_{0}}
$$

Note that $\epsilon_{k_{0}} \geq\left\|\tilde{h}_{k_{0}}\right\|^{2}$, then by choosing $\delta_{k_{0}}=-\tilde{h}_{k_{0}}$, we have $\left\|\delta_{k_{0}}\right\|=\left\|\widetilde{h}_{k_{0}}\right\| \leq \epsilon_{k_{0}}$. So we derive an instance $h_{k_{0}}^{\prime}=\delta_{k_{0}}+\widetilde{h}_{k_{0}}=$ 0 with $h_{k_{0}}^{\prime} \in U_{k_{0}}$. Substituting $h_{k_{0}}^{\prime}=0$ into the left side of the above inequality, it is easy to result in a contradiction.

Obviously, the same results as those of Lemma 1 hold for (RP).

\section{SDP Relaxation for Problem (RP)}

In this section, we will present the SDP relaxation problem denoted by (SDR) for handling problem (RP) efficiently. We adopt the same formulation of the SDP relaxation with that in [17]. Then some properties for (SDR) will be explored, especially the properties at the optimal solution. In the next section, based on these properties, we will show that (SDR) will yield rank-one solutions under proper assumption and then provide globally optimal solutions of (RP).

In the previous section, a convex relaxation programming (RPR) is derived. Note that the (RPR) problem has an infinite number of linear inequality constraints. Such an infinite number of inequalities can be turned to a finite number of linear matrix inequalities (LMIs), by using the following Sprocedure $[19,20]$.

Lemma 2 (S-procedure). Let $A, C \in H^{n \times n}$ be complex Hermitian matrices, and $b \in C^{n}, c \in R$. The following condition

$$
e^{H} A e+b^{H} e+e^{H} b+c \geq 0, \quad \forall e^{H} C e \leq 1,
$$

holds if and only if there exists a $\lambda \geq 0$ such that

$$
\left[\begin{array}{cc}
A+\lambda C & b \\
b^{H} & c-\lambda
\end{array}\right] \geq 0 .
$$

By applying Lemma 2 to the constraints in (RPR), one can reformulate problem (RPR) equivalently as

$$
\begin{array}{ll}
\min _{\left\{\alpha_{k}\right\}_{k=1}^{K},\left\{g_{k}\right\}_{k=1}^{K}} & \sum_{k=1}^{K} \operatorname{Tr}\left(G_{k}\right) \\
\text { s.t. } & \alpha_{k} \geq 0 \\
& {\left[\begin{array}{cc}
X_{k}+\alpha_{k} I & X_{k} \tilde{h}_{k} \\
\tilde{h}_{k}^{H} X_{k}^{H} & \widetilde{h}_{k}^{H} X_{k} \widetilde{h}_{k}-\sigma_{k}^{2}-\alpha_{k} \epsilon_{k}^{2}
\end{array}\right] \geq 0,} \\
& G_{k} \geq 0, \quad k=1, \ldots, K,
\end{array}
$$

where $X_{k}=\left(1 / \gamma_{k}\right) G_{k}-\sum_{i \neq k} G_{i}$. One can see that problem (SDR) has a linear objective function and convex constraints. So the problem (SDR) is a SDP relaxation to the problem (RP) and also is a convex SDP problem. This means that any local minima must be a global minima and the set of optimal solutions is convex. It is known that the problem (SDR) can be efficiently solved by CVX, SeDuMi, or YALMIP solvers.

Before we finish this section, we explore some more properties of (RPR) or (SDR) (noting that these two problems are equivalent), especially at the optimal solution. Define

$$
f_{k}\left(\delta_{k}\right)=\left(\widetilde{h}_{k}+\delta_{k}\right)^{H}\left(\frac{1}{\gamma_{k}} G_{k}-\sum_{i \neq k} G_{i}\right)\left(\widetilde{h}_{k}+\delta_{k}\right)-\sigma_{k}^{2} .
$$

Equivalently, we have

$$
f_{k}\left(\delta_{k}\right)=\left[\begin{array}{ll}
\delta_{k}^{H} & 1
\end{array}\right]\left[\begin{array}{cc}
X_{k} & X_{k} \widetilde{h}_{k} \\
\widetilde{h}_{k}^{H} X_{k}^{H} & \widetilde{h}_{k}^{H} X_{k} \widetilde{h}_{k}-\sigma_{k}^{2}
\end{array}\right]\left[\begin{array}{cc}
\delta_{k}^{H} & 1
\end{array}\right]^{T} .
$$

By reformulating the constraints in (RPR), we can see that the constraint in (RPR) is equivalent to

$$
f_{k}\left(\delta_{k}\right) \geq 0, \quad \forall\left\|\delta_{k}\right\|^{2} \leq \epsilon_{k}^{2} .
$$

For simplification during the proof, denote

$$
P_{k}=\left[\begin{array}{cc}
X_{k}+\alpha_{k} I & X_{k} \tilde{h}_{k} \\
\widetilde{h}_{k}^{H} X_{k}^{H} & \widetilde{h}_{k}^{H} X_{k} \widetilde{h}_{k}-\sigma_{k}^{2}-\alpha_{k} \epsilon_{k}^{2}
\end{array}\right] .
$$

Also define

$$
f_{k}^{\prime}\left(\delta_{k}\right)=\left[\begin{array}{ll}
\delta_{k}^{H} & 1
\end{array}\right] P_{k}\left[\begin{array}{c}
\delta_{k} \\
1
\end{array}\right]
$$

that is,

$$
\begin{aligned}
f_{k}^{\prime}\left(\delta_{k}\right)= & \delta_{k}^{H}\left(X_{k}+\alpha_{k} I\right) \delta_{k}+2 \widetilde{h}_{k}^{H} X_{k} \delta_{k}+\widetilde{h}_{k}^{H} X_{k} \widetilde{h}_{k} \\
& -\sigma_{k}^{2}-\alpha_{k} \epsilon_{k}^{2} .
\end{aligned}
$$

According to the definition $f_{k}\left(\delta_{k}\right)$ in (8), we have

$$
f_{k}^{\prime}\left(\delta_{k}\right)=f_{k}\left(\delta_{k}\right)+\alpha_{k}\left(\left\|\delta_{k}\right\|^{2}-\epsilon_{k}^{2}\right) .
$$

In the following lemma, it will be shown that at the optimal solution for $k=1, \ldots, K$ there must exist $\delta_{k}^{*}$ such that $f_{k}\left(\delta_{k}^{*}\right)=0$ and $\left\|\delta_{k}^{*}\right\|^{2}=\epsilon_{k}^{2}$, which implies that $f_{k}^{\prime}\left(\delta_{k}^{*}\right)=0$ according to (14).

Lemma 3. Let $\left\{\alpha_{k}\right\},\left\{G_{k}\right\}$ be any optimal solutions of (SDR). Then for each $k=1, \ldots, K$, there must exist $\delta_{k}^{*}$ such that $\left(\widetilde{h}_{k}+\right.$ $\left.\delta_{k}^{*}\right)^{H} G_{k}\left(\tilde{h}_{k}+\delta_{k}^{*}\right) /\left(\sum_{i \neq k}\left(\tilde{h}_{k}+\delta_{k}^{*}\right)^{H} G_{i}\left(\tilde{h}_{k}+\delta_{k}^{*}\right)+\sigma_{k}^{2}\right)=\gamma_{k}$, where $\left\|\delta_{k}^{*}\right\|=\epsilon_{k}$.

Proof. First we will show at the optimal solution there exist $\delta_{k}^{*}$ such that

$$
\frac{\left(\widetilde{h}_{k}+\delta_{k}^{*}\right)^{H} G_{k}\left(\widetilde{h}_{k}+\delta_{k}^{*}\right)}{\sum_{i \neq k}\left(\widetilde{h}_{k}+\delta_{k}^{*}\right)^{H} G_{i}\left(\widetilde{h}_{k}+\delta_{k}^{*}\right)+\sigma_{k}^{2}}=\gamma_{k}
$$


and $\left\|\delta_{k}^{*}\right\| \leq \epsilon_{k}$. On the contrary, assume that there exists an index $j$ such that

$$
\frac{h_{j}^{H} G_{j} h_{j}}{\sum_{i \neq j} h_{j}^{H} G_{i} h_{j}+\sigma_{j}^{2}}>\gamma_{j}, \quad \forall h_{j} \in U_{j}=\left\{\widetilde{h}_{j}+\delta_{j} \mid\left\|\delta_{j}\right\| \leq \epsilon_{j}\right\} .
$$

Note that $U_{j}$ is a compact set, then there must exist $\widehat{h}_{j} \in$ $U_{j}$ such that at this instance, the ratio function $h_{j}^{H} G_{j} h_{j} /$ $\left(\sum_{i \neq j} h_{j}^{H} G_{i} h_{j}+\sigma_{j}^{2}\right)$ can attain its minimum over the compact set $U_{j}$. From (16), this implies that

$$
\frac{\widehat{h}_{j}^{H} G_{j} \widehat{h}_{j}}{\sum_{i \neq j} \widehat{h}_{j}^{H} G_{i} h_{j}+\sigma_{j}^{2}}=\min _{h_{j} \in U_{j}} \frac{h_{j}^{T} G_{j} h_{j}}{\sum_{i \neq j} h_{j}^{H} G_{i} h_{j}+\sigma_{j}^{2}}>\gamma_{j} .
$$

Let $\widehat{h}_{j}^{T} G_{j} \widehat{h}_{j} /\left(\sum_{i \neq j} \widehat{h}_{j}^{H} G_{i} h_{j}+\sigma_{j}^{2}\right)=t_{j} \gamma_{j}$, then obviously $t_{j}>1$. Then, a new point $\left\{G_{k}^{\prime}\right\}$ will be constructed as follows:

$$
G_{k}^{\prime}= \begin{cases}G_{k}^{\prime}=\frac{1}{t_{j}} G_{k}, & k=j, \\ G_{k}^{\prime}=G_{k}, & k \neq j .\end{cases}
$$

Next we will show the feasibility of $\left\{G_{k}^{\prime}\right\}$ in (RPR). Obviously $G_{k}^{\prime} \geq 0$, so it is just necessary to show that for $k=1, \ldots, K$, the following inequalities hold in (RPR):

$$
\frac{h_{k}^{H} G_{k}^{\prime} h_{k}}{\sum_{i \neq k} h_{k}^{H} G_{i}^{\prime} h_{k}+\sigma_{k}^{2}} \geq \gamma_{k}, \quad \forall h_{k} \in U_{k} .
$$

For $k=j$, according to the definition of $t_{j}$, it holds that for all $h_{k} \in U_{k}$,

$$
\frac{h_{k}^{H} G_{k}^{\prime} h_{k}}{\sum_{i \neq k} h_{k}^{H} G_{i}^{\prime} h_{k}+\sigma_{k}^{2}}=\frac{\left(1 / t_{k}\right) h_{k}^{H} G_{k} h_{k}}{\sum_{i \neq k} h_{k}^{H} G_{i} h_{k}+\sigma_{k}^{2}} \geq \gamma_{k} .
$$

For $k \neq j$, it holds that for all $h_{k} \in U_{k}$,

$$
\frac{h_{k}^{H} G_{k}^{\prime} h_{k}}{\sum_{i \neq k} h_{k}^{H} G_{i}^{\prime} h_{k}+\sigma_{k}^{2}} \geq \frac{h_{k}^{H} G_{k} h_{k}}{\sum_{i \neq k} h_{k}^{H} G_{i} h_{k}+\sigma_{k}^{2}} \geq \gamma_{k} .
$$

The above discussion shows that $G_{k}^{\prime}$ is a feasible point of (RPR). Also from the construction of $\left\{G_{k}^{\prime}\right\}$, it is easy to see that $\sum_{k=1}^{K} \operatorname{Tr}\left(G_{k}^{\prime}\right)<\sum_{k=1}^{K} \operatorname{Tr}\left(G_{k}\right)$, which contradicts the assumption that $\left\{G_{k}\right\}$ is the optimal solution to (RPR). So, for any solution $\left\{G_{k}\right\}$, for each $k=1, \ldots, K$, there exist a $\delta_{k}^{*} \in C^{M}$ such that

$$
\frac{\left(\widetilde{h}_{k}+\delta_{k}^{*}\right)^{H} G_{k}\left(\widetilde{h}_{k}+\delta_{k}^{*}\right)}{\sum_{i \neq k}\left(\widetilde{h}_{k}+\delta_{k}^{*}\right)^{H} G_{i}\left(\widetilde{h}_{k}+\delta_{k}^{*}\right)+\sigma_{k}^{2}}=\gamma_{k}
$$

which also means that $f_{k}\left(\delta_{k}^{*}\right)=0$.

Next we just need to show $\left\|\delta_{k}^{*}\right\|=\epsilon_{k}$. Note that the problem (RPR) is equivalent to the problem (SDR); then the solution $\left\{\alpha_{k}\right\},\left\{G_{k}\right\}$ is also the solution of (SDR). So we have $P_{k} \geq 0$. Then it holds that

$$
\begin{aligned}
f_{k}^{\prime}\left(\delta_{k}^{*}\right) & =\left[\begin{array}{ll}
\delta_{k}^{* H} & 1
\end{array}\right] P_{k}\left[\begin{array}{ll}
\delta_{k}^{* H} & 1
\end{array}\right]^{H} \\
& =f_{k}\left(\delta_{k}^{*}\right)+\alpha_{k}\left(\left\|\delta_{k}^{*}\right\|^{2}-\epsilon_{k}^{2}\right) \geq 0 .
\end{aligned}
$$

Noting the fact that $f_{k}\left(\delta_{k}^{*}\right)=0$, we have $\alpha_{k}\left\|\delta_{k}^{*}\right\|^{2}-\alpha_{k} \epsilon_{k}^{2} \geq 0$. Since $\left\|\delta_{k}^{*}\right\|^{2} \leq \epsilon_{k}^{2}, \alpha_{k} \geq 0$, then we can get

$$
\alpha_{k}\left\|\delta_{k}^{*}\right\|^{2}-\alpha_{k} \epsilon_{k}^{2}=0 .
$$

If $\alpha_{k}=0$, noting the fact that $P_{k} \geq 0$, we have

$$
\left[\begin{array}{cc}
X_{k} & X_{k} \tilde{h}_{k} \\
\widetilde{h}_{k}^{H} X_{k}^{H} & \widetilde{h}_{k}^{H} X_{k} \widetilde{h}_{k}-\sigma_{k}^{2}
\end{array}\right] \geq 0 .
$$

Note that

$$
f_{k}\left(\delta_{k}^{*}\right)=\left[\begin{array}{ll}
\delta_{k}^{* T} & 1
\end{array}\right]\left[\begin{array}{cc}
X_{k} & X_{k} \widetilde{h}_{k} \\
\widetilde{h}_{k}^{H} X_{k}^{H} & \widetilde{h}_{k}^{H} X_{k} \widetilde{h}_{k}-\sigma_{k}^{2}
\end{array}\right]\left[\begin{array}{cc}
\delta_{k}^{* T} & 1
\end{array}\right]^{T}=0 .
$$

Then

$$
\left[\begin{array}{cc}
X_{k} & X_{k} \widetilde{h}_{k} \\
\widetilde{h}_{k}^{H} X_{k}^{H} & \widetilde{h}_{k}^{H} X_{k} \widetilde{h}_{k}-\sigma_{k}^{2}
\end{array}\right]\left[\begin{array}{c}
\delta_{k}^{*} \\
1
\end{array}\right]=0 .
$$

It follows that $X_{k}\left(\delta_{k}^{*}+\widetilde{h}_{k}\right)=0$ which means that

$$
f_{k}\left(\delta_{k}^{*}\right)=\left(\delta_{k}^{*}+\widetilde{h}_{k}\right)^{H} X_{k}\left(\delta_{k}^{*}+\widetilde{h}_{k}\right)-\sigma_{k}^{2}=-\sigma_{k}^{2}<0 .
$$

This contradiction shows that $\alpha_{k}>0$. Then, from (24), we have that $\left\|\delta_{k}^{*}\right\|=\epsilon_{k}$. This completes the proof.

Note that the vector $\delta_{k}^{*}$ may not be unique; then we can collect all these vectors as a set $\Lambda_{k}$ where

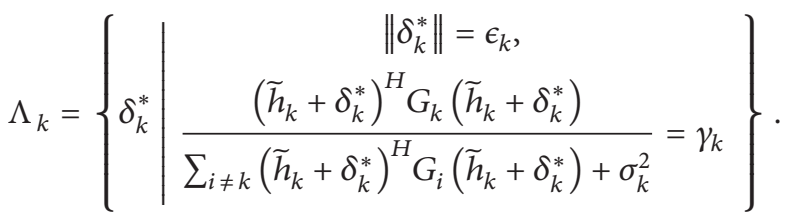

From Lemma 3, since at $\delta_{k}^{*}$, the constraint is active, then the function value $f_{k}\left(\delta_{k}\right)$ at point $\delta_{k}^{*}$ is zero; that is,

$$
f_{k}\left(\delta_{k}^{*}\right)=\delta_{k}^{* H} X_{k} \delta_{k}^{*}+2 h_{k}^{T} X_{k} \delta_{k}^{*}+h_{k}^{H} X_{k} h_{k}-\sigma_{k}^{2}=0 .
$$

So we can conclude that at any solution $\left\{G_{k}\right\},\left\{\alpha_{k}\right\}$ of (SDR), any point in the set $\Lambda_{k}$ must be the optimal solution of the following nonconvex quadratic programming $\left(Q P_{k}\right)$ where $k=1, \ldots, K$ :

$\min f_{k}\left(\delta_{k}\right)=\left[\begin{array}{ll}\delta_{k}^{H} & 1\end{array}\right]\left[\begin{array}{cc}X_{k} & X_{k} \widetilde{h}_{k} \\ \widetilde{h}_{k}^{H} X_{k}^{H} & \widetilde{h}_{k}^{H} X_{k} \widetilde{h}_{k}-\sigma_{k}^{2}\end{array}\right]\left[\begin{array}{cc}\delta_{k}^{H} & 1\end{array}\right]^{T}$

s.t. $\left\|\delta_{k}\right\|^{2}=\epsilon_{k}^{2}$, 
where $X_{k}=\left(G_{k} / \gamma_{k}\right)-\sum_{i \neq k} G_{i}$ and the optimal value of $\left(Q P_{k}\right)$ is 0 .

In the above lemma, we just discuss the existence of $\delta_{k}^{*}$. In the following lemma, we will explore more properties about these vectors.

Lemma 4. Let $\left\{\alpha_{k}\right\},\left\{G_{k}\right\}$ be any optimal solutions of (SDR); then it holds that $\operatorname{rank}\left(X_{k}+\alpha_{k} I\right)=\operatorname{rank} P_{k}$, and for any $\delta_{k}^{*} \in$ $\Lambda_{k}^{*}, \delta_{k}^{*}$ is not in the null space of $X_{k}+\alpha_{k} I$.

Proof. Let $\left\{\alpha_{k}\right\},\left\{G_{k}\right\}$ be any optimal solutions of (SDR); then $P_{k} \geq 0$. From Lemma 3 , it is easy to see that for any $\delta_{k}^{*} \in \Lambda_{k}$,

$$
\begin{aligned}
f_{k}^{\prime}\left(\delta_{k}^{*}\right) & =\left[\begin{array}{ll}
\delta_{k}^{* H} & 1
\end{array}\right] P_{k}\left[\begin{array}{ll}
\delta_{k}^{* H} & 1
\end{array}\right]^{H} \\
& =f_{k}\left(\delta_{k}^{*}\right)+\alpha_{k}\left(\left\|\delta_{k}^{*}\right\|^{2}-\epsilon_{k}^{2}\right)=0 .
\end{aligned}
$$

Then, it holds that $\left[\begin{array}{ll}\delta_{k}^{* H} & 1\end{array}\right] P_{k}=0$, which implies that the last row $\left[\widetilde{h}_{k}^{H} X_{k}^{H} \widetilde{h}_{k}^{H} X_{k} \widetilde{h}_{k}-\sigma_{k}^{2}-\alpha_{k} \epsilon_{k}^{2}\right]$ in the matrix $P_{k}$ can be linearly expressed by the row vectors of the matrix $\left[\begin{array}{ll}X_{k}+\alpha_{k} I & X_{k} \widetilde{h}_{k}\end{array}\right]$, so $\operatorname{rank} P_{k}=\operatorname{rank}\left[X_{k}+\alpha_{k} I X_{k} \widetilde{h}_{k}\right]$. Also, noting that $P_{k}\left[\begin{array}{c}\delta_{k}^{*} \\ 1\end{array}\right]=0, X_{k} \widetilde{h}_{k}$ can be linearly expressed by the column vectors of $X_{k}+\alpha_{k} I$, so

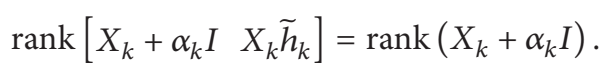

Then, we have $\operatorname{rank}\left(X_{k}+\alpha_{k} I\right)=\operatorname{rank} P_{k}$.

Next we will prove the second conclusion of this lemma. On the contrary, if there exists $\delta_{k}^{*} \in \Lambda_{k}$ which is in the null space of $X_{k}+\alpha_{k} I$, then from $P_{k}\left[\begin{array}{c}\delta_{k}^{*} \\ 1\end{array}\right]=0$, it holds that $X_{k} \widetilde{h}_{k}=$ 0 which implies that

$$
\widetilde{h}_{k}^{H} X_{k} \widetilde{h}_{k}-\sigma_{k}^{2}-\alpha_{k} \epsilon_{k}^{2}=-\sigma_{k}^{2}-\alpha_{k} \epsilon_{k}^{2}<0 .
$$

This contradicts the fact $P_{k} \geq 0$.

The main goal of the next two lemmas is to construct a basis of the null space of the matrix $P_{k}(k=1, \ldots, K)$ related to the optimal solution. These results will be prepared for proving the tightness of the (SDR) in the next section.

Lemma 5. Let $\left\{\alpha_{k}\right\},\left\{G_{k}\right\}$ be any optimal solutions of (SDR), and let the vectors $\beta_{1}^{k}, \beta_{2}^{k}, \ldots, \beta_{t_{k}}^{k}$ be a basis for the null space of $X_{k}+\alpha_{k} I$; then for any $\delta_{k}^{*} \in \Lambda_{k}$, the set of vectors

$$
\left[\begin{array}{c}
\delta_{k}^{\star} \\
1
\end{array}\right],\left[\begin{array}{c}
\beta_{1}^{k} \\
0
\end{array}\right], \ldots,\left[\begin{array}{c}
\beta_{t_{k}}^{k} \\
0
\end{array}\right]
$$

forms a basis for the null space of the matrix $P_{k}(k=1, \ldots, K)$.

Proof. From Lemma 3, for any $\delta_{k}^{*} \in \Lambda_{k}$, we have $f_{k}\left(\delta_{k}^{*}\right)=0$ and $f_{k}^{\prime}\left(\delta_{k}^{*}\right)=0$. Noting that $P_{k} \succeq 0$, then for $k=1, \ldots, K, \delta_{k}^{*}$ must be the optimal solution to the convex programming

$$
\min f_{k}^{\prime}\left(\delta_{k}\right) \text {. }
$$

Then

$$
\nabla f_{k}^{\prime}\left(\delta_{k}^{*}\right)=\left(X_{k}+\alpha_{k} I\right) \delta_{k}^{*}+X_{k} \tilde{h}_{k}=0
$$

From the assumption that the set of vectors $\beta_{1}^{k}, \beta_{2}^{k}, \ldots, \beta_{t_{k}}^{k}$ forms a basis for the null space of $X_{k}+\alpha_{k} I$, then

$$
\left(X_{k}+\alpha_{k} I\right) \beta_{i}^{k}=0, \quad i=1, \ldots, t_{k} .
$$

From (37), we have

$$
\left(X_{k}+\alpha_{k} I\right)\left(\beta_{i}^{k}+\delta_{k}^{*}\right)+X_{k} \widetilde{h}_{k}=0, \quad i=1, \ldots, t_{k}
$$

that is,

$$
\nabla f_{k}^{\prime}\left(\beta_{i}^{k}+\delta_{k}^{*}\right)=\left(X_{k}+\alpha_{k} I\right)\left(\beta_{i}^{k}+\delta_{k}^{*}\right)+X_{k} \widetilde{h}_{k}=0 .
$$

From the optimality condition for convex programming (36), the vectors $\beta_{i}^{k}+\delta_{k}^{*}\left(i=1, \ldots, t_{k}\right)$ are also the optimal solutions for (36); that is,

$$
f_{k}^{\prime}\left(\beta_{i}^{k}+\delta_{k}^{*}\right)=0, \quad i=1, \ldots, t_{k} .
$$

Since $P_{k} \succeq 0$, then

$$
P_{k}\left[\begin{array}{c}
\beta_{i}^{k}+\delta_{k}^{*} \\
1
\end{array}\right]=0
$$

which implies that

$$
P_{k}\left[\begin{array}{c}
\beta_{i}^{k}+\delta_{k}^{*} \\
1
\end{array}\right]-P_{k}\left[\begin{array}{c}
\delta_{k}^{*} \\
1
\end{array}\right]=P_{k}\left[\begin{array}{c}
\beta_{i}^{k} \\
0
\end{array}\right]=0 .
$$

From Lemma 4, it is known that $\delta_{k}^{*}$ is not in the null space, then the family of vectors $\delta_{k}^{*}, \beta_{1}, \ldots, \beta_{t_{k}}$ is linearly independent, which also means that the family of vectors (35) is linearly independent. From Lemma $4, \operatorname{rank}\left(X_{k}+\alpha_{k} I\right)=$ $M-t_{k}=\operatorname{rank}\left(P_{k}\right)$. So the dimension of the null space of $P_{k}$ is $M+1-\operatorname{rank}\left(P_{k}\right)=t_{k}+1$. Then, we can conclude that the set of vectors (35) is a basis for the null space of $P_{k}$.

In the above Lemma 5, we construct a basis for the null space of $P_{k}$; then in the next lemma, under proper assumption, we can derive an orthogonal basis for the null space of $P_{k}$ related to any optimal solution. Let $\left\{\alpha_{k}\right\},\left\{G_{k}\right\}$ be any optimal solutions of (SDR). Before we present the lemma, the following assumption will be given.

Assumption $A$. There exists a unique $\delta_{k}^{*}$ such that $\left\|\delta_{k}^{*}\right\|=\epsilon_{k}$ and

$$
\frac{\left(\widetilde{h}_{k}+\delta_{k}^{*}\right)^{H} G_{k}\left(\widetilde{h}_{k}+\delta_{k}^{*}\right)}{\sum_{i \neq k}\left(\widetilde{h}_{k}+\delta_{k}^{*}\right)^{H} G_{i}\left(\widetilde{h}_{k}+\delta_{k}^{*}\right)+\sigma_{k}^{2}}=\gamma_{k} .
$$

Note that in Lemma 3, we have proved the existence of $\delta_{k}^{*}$ which satisfies the conditions in Assumption A. But in Assumption A, we restrict that the $\delta_{k}^{*}$ is unique. Equivalently, Assumption A can be rewritten as the following Assumption B.

Assumption B. Assume that the set $\Lambda_{k}$ is a single point. Also, we have Assumption $C$ equivalently. 
Assumption $C$. Assume that for any $k=1, \ldots, K$, the following nonconvex quadratic programming with one equality constraint has unique solution:

$$
\begin{array}{ll}
\min & f_{k}\left(\delta_{k}\right)=\left[\begin{array}{ll}
\delta_{k}^{H} & 1
\end{array}\right]\left[\begin{array}{cc}
X_{k} & X_{k} \widetilde{h}_{k} \\
\widetilde{h}_{k}^{H} X_{k}^{H} & \widetilde{h}_{k}^{H} X_{k} \widetilde{h}_{k}-\sigma_{k}^{2}
\end{array}\right]\left[\begin{array}{c}
\delta_{k} \\
1
\end{array}\right] . \\
\text { s.t. } & \left\|\delta_{k}\right\|^{2}=\epsilon_{k}^{2} .
\end{array}
$$

Note that the above Assumptions A, B, and C are equivalent. Under any assumption, we can construct an orthogonal basis for $P_{k}$. This result is described in the following lemma.

Lemma 6. Let $\left\{\alpha_{k}\right\},\left\{G_{k}\right\}$ be any optimal solutions of (SDR). Assume that Assumption A holds, and let the set of vectors $\beta_{1}^{k}, \beta_{2}^{k}, \ldots, \beta_{t_{k}}^{k}$ be an orthogonal basis for the null space of $X_{k}+\alpha_{k} I$; then the set of vectors $\left[\begin{array}{c}\delta_{k}^{\star} \\ 1\end{array}\right],\left[\begin{array}{c}\beta_{1}^{k} \\ 0\end{array}\right], \ldots,\left[\begin{array}{c}\beta_{t_{k}}^{k} \\ 0\end{array}\right]$ must be an orthogonal basis for the null space of $P_{k}$.

Proof. From Lemma 5, it is easy to see that the set of vectors $\left[\begin{array}{c}\delta_{k}^{\star} \\ 1\end{array}\right],\left[\begin{array}{c}\beta_{1}^{k} \\ 0\end{array}\right], \ldots,\left[\begin{array}{c}\beta_{t_{k}}^{k} \\ 0\end{array}\right]$ is a basis for the null space of $P_{k}$. Noting that the set of vectors $\beta_{1}^{k}, \beta_{2}^{k}, \ldots, \beta_{t_{k}}^{k}$ is an orthogonal basis for the null space of $X_{k}+\alpha_{k} I$, it is just necessary to show that $\delta_{k}^{\star}$ is orthogonal to $\beta_{j}^{k}\left(j=1, \ldots, t_{k}\right)$. On the contrary, if $\delta_{k}^{\star}$ is not orthogonal to $\beta_{j}^{k}$, then let $l=-2 \delta_{k}^{* H} \beta_{j}^{k} /\left\|\beta_{j}^{k}\right\|^{2}$. Obviously $l \neq 0$. It is also easy to check that

$$
\left\|\delta_{k}^{*}+l \beta_{j}^{k}\right\|^{2}=\left(\delta_{k}^{*}+l \beta_{j}^{k}\right)^{H}\left(\delta_{k}^{*}+l \beta_{j}^{k}\right)=\epsilon_{k}^{2} .
$$

From the proof of Lemma 5, it holds that

$$
P_{k}\left[\begin{array}{c}
\delta_{k}^{*}+l \beta_{j}^{k} \\
1
\end{array}\right]=0
$$

then we have

$$
\left[\left(\delta_{k}^{*}+l \beta_{j}^{k}\right)^{H} 1\right] P_{k}\left[\begin{array}{c}
\delta_{k}^{*}+l \beta_{j}^{k} \\
1
\end{array}\right]=0 .
$$

So $f_{k}^{\prime}\left(\delta_{k}^{*}+l \beta_{j}^{k}\right)=\left[\left(\delta_{k}^{*}+l \beta_{j}^{k}\right)^{H} 1\right] P_{k}\left[\begin{array}{c}\delta_{k}^{*}+l \beta_{j}^{k} \\ 1\end{array}\right]=0$. From (46), we have $f_{k}\left(\delta_{k}^{*}+l \beta_{j}^{k}\right)=0$, which contradicts Assumption A. Then, $\delta_{k}^{*}$ must be orthogonal to $\beta_{i}^{k}\left(i=1, \ldots, t_{k}\right)$. This completes the proof.

\section{Tightness of SDR}

In order to prove the tightness of (SDR), we need to pay more attention to the dual of the problem (SDR). So firstly we write down the dual of the problem (SDR) denoted by (D-SDR) as follows:

$$
\begin{array}{ll}
\max _{\left\{\lambda_{k}\right\}_{k=1}^{K},\left\{y_{k}\right\}_{k=1}^{K},\left\{Y_{k}\right\}_{k=1}^{K}} & \sum_{k=1}^{K} \lambda_{k} \sigma_{k}^{2} \\
\text { s.t. } & \lambda_{k} \epsilon_{k}^{2}-\operatorname{Tr}\left(Y_{k}\right) \geq 0, \\
& {\left[\begin{array}{cc}
Y_{k} & y_{k} \\
y_{k}^{H} & \lambda_{k}
\end{array}\right] \geq 0 .} \\
& Z_{k}=I-\frac{1}{\gamma_{k}} \\
& \times\left(Y_{k}+y_{k} \widetilde{h}_{k}^{H}+\widetilde{h}_{k} y_{k}^{H}+\lambda_{k} \widetilde{h}_{k} \widetilde{h}_{k}^{H}\right) \\
& +\sum_{i \neq k}\left(Y_{i}+y_{i} \widetilde{h}_{i}^{H}+\widetilde{h}_{i} y_{i}^{H}+\lambda_{i} \widetilde{h}_{i} \widetilde{h}_{i}^{H}\right) \\
& \\
& 0, \quad k=1, \ldots, K .
\end{array}
$$

From [17], one can see that the dual problem (D-SDR) has the following properties.

Proposition 7. There always exists strictly feasible point for problem (D-SDR).

From Proposition 7, the feasible region of (D-SDR) has nonempty interior. If, in addition, the primal (SDR) also has nonempty interior, then the following strong duality holds between (SDR) and (D-SDR). Let $z_{P}^{*}$ and $z_{D}^{*}$ denote the optimal objective function values of (SDR) and (D-SDR). Suppose that there exists a strict feasible point for (SDR). Then both (SDR) and (D-SDR) attain their optimal values, and $z_{P}^{*}=z_{D}^{*}$.

From the optimality conditions of convex programming, assume that the problem (SDR) is strictly feasible; then $\left\{G_{k}\right\},\left\{\alpha_{k}\right\}$ is the solution of (SDR) if and only if there exist $\left\{U_{k}\right\},\left\{Z_{k}\right\},\left\{\lambda_{k}\right\}$ satisfying the following KKT condition:

$$
\begin{gathered}
G_{k} \geq 0, \\
P_{k}=\left[\begin{array}{cc}
X_{k}+\alpha_{k} I & X_{k} \tilde{h}_{k} \\
\tilde{h}_{k}^{H} X_{k}^{H} & \tilde{h}_{k}^{H} X_{k} \widetilde{h}_{k}-\sigma_{k}^{2}-\alpha_{k} \epsilon_{k}^{2}
\end{array}\right] \geq 0, \\
\alpha_{k} \geq 0, \quad U_{k}=\left[\begin{array}{cc}
Y_{k} & y_{k} \\
y_{k}^{H} & \lambda_{k}
\end{array}\right] \geq 0, \\
Z_{k}=I-\frac{1}{\gamma_{k}}\left(Y_{k}+y_{k} \tilde{h}_{k}^{H}+\tilde{h}_{k} y_{k}^{H}+\lambda_{k} \tilde{h}_{k} \tilde{h}_{k}^{H}\right) \\
+\sum_{i \neq k}\left(Y_{i}+y_{i} \widetilde{h}_{i}^{H}+\tilde{h}_{i} y_{i}^{H}+\lambda_{i} \widetilde{h}_{i} \widetilde{h}_{i}^{H}\right) \geq 0, \\
\lambda_{k} \epsilon_{k}^{2}-\operatorname{Tr}\left(Y_{k}\right) \geq 0,
\end{gathered}
$$




$$
\begin{gathered}
\alpha_{k}\left(\lambda_{k} \epsilon_{k}^{2}-\operatorname{Tr}\left(Y_{k}\right)\right)=0, \\
\operatorname{Tr}\left(P_{k} U_{k}\right)=0, \quad \operatorname{Tr}\left(G_{k} Z_{k}\right)=0 .
\end{gathered}
$$

Based on the above results, under proper assumption, we can show the tightness of the problem (SDR) in the following theorem.

Theorem 8. Let $\left\{G_{k}\right\},\left\{\alpha_{k}\right\}$ be the optimal solution to (SDR). Assume that the problem (SDR) is strictly feasible and Assumption $A$ holds; then $\left\{G_{k}\right\}$ must be rank one.

Proof. From Lemma 6, we have known that the set of vectors $\left[\begin{array}{c}\delta_{k}^{*} \\ 1\end{array}\right],\left[\begin{array}{c}\beta_{1}^{k} \\ 0\end{array}\right], \ldots,\left[\begin{array}{c}\beta_{t_{k}}^{k} \\ 0\end{array}\right]$ is the orthogonal basis for the null space of $P_{k}$ where the set of vectors $\left[\begin{array}{c}\beta_{1}^{k} \\ 0\end{array}\right], \ldots,\left[\begin{array}{c}\beta_{t_{k}}^{k} \\ 0\end{array}\right]$ is the orthogonal basis for the null space of $X_{k}+\alpha_{k} I$. From the complementarity slackness conditions in the KKT condition, it holds that $\operatorname{Tr}\left(P_{k} U_{k}\right)=0$. Since $U_{k} \geq 0$, then obviously $U_{k}$ can be expressed as $U_{k}=E \Psi_{k} E^{H}$, where $\Psi_{k}=\left\{\psi_{i j}\right\}_{\left(t_{k}+1\right) \times\left(t_{k}+1\right)}$, and

$$
E=\left[\begin{array}{c}
E_{m} \\
e
\end{array}\right], \quad E_{m}=\left[\begin{array}{lllll}
\delta_{k}^{*} & \beta_{1}^{k} & \beta_{2}^{k} & \cdots & \beta_{t_{k}}^{k}
\end{array}\right],
$$

$e=\left[\begin{array}{lllll}1 & 0 & 0 & \cdots & 0\end{array}\right]$. So it follows that

$$
U_{k}=\left[\begin{array}{cc}
E_{m} \Psi_{k} E_{m}^{H} & E_{m} \Psi_{k} e \\
e \Psi_{k} E_{m}^{H} & e \Psi_{k} e^{H}
\end{array}\right]=\left[\begin{array}{cc}
Y_{k} & y_{k} \\
y_{k}^{H} & \lambda_{k}
\end{array}\right]
$$

Obviously, $\lambda_{k}=e \Psi_{k} e^{H}=\psi_{11}$, and $Y_{k}=E_{m} \Psi_{k} E_{m}$. Noting that the vectors $\delta_{k}^{*}, \beta_{1}^{k}, \ldots, \beta_{t_{k}}^{k}$ are orthogonal, then it holds that $\operatorname{Tr}\left(Y_{k}\right)=\operatorname{Tr}\left(E_{m} \Psi_{k} E_{m}^{H}\right)=\operatorname{Tr}\left(\Psi_{k} E_{m}^{H} E_{m}\right)=$ $\lambda_{k} \epsilon_{k}^{2}+\sum_{i=2}^{t_{k}+1} \psi_{i i}\left\|\beta_{i-1}^{k}\right\|^{2}$. From the KKT condition, since $\lambda_{k} \epsilon_{k}^{2}-$ $\operatorname{Tr}\left(Y_{k}\right) \geq 0$, then it must hold that $\psi_{i i}=0, i \geq 2$. Noting that $\Psi_{k}$ is positive semidefinite, then it is easy to derive the concrete expression of $\Psi_{k}$ as follows:

$$
\Psi_{k}=\left[\begin{array}{cccc}
\lambda_{k} & 0 & \cdots & 0 \\
0 & 0 & \cdots & 0 \\
\vdots & \vdots & \ddots & 0 \\
0 & 0 & 0 & 0
\end{array}\right],
$$

which implies that $Y_{k}=\lambda_{k} \delta_{k}^{*} \delta_{k}^{* H}$ and $U_{k}=\left[\begin{array}{cc}\lambda_{k} \delta_{k}^{*} \delta_{k}^{* H} & \lambda_{k} \delta_{k}^{*} \\ \lambda_{k} \delta_{k}^{* H} & \lambda_{k}\end{array}\right]$. Then, the matrix $Z_{k}$ can be expressed in the following form:

$$
\begin{aligned}
Z_{k}= & I-\frac{1}{\gamma_{k}} \lambda_{k}\left(\widetilde{h}_{k}+\delta_{k}^{*}\right)\left(\widetilde{h}_{k}+\delta_{k}^{*}\right)^{H} \\
& +\sum_{i \neq k} \lambda_{i}\left(\widetilde{h}_{i}+\delta_{i}^{*}\right)\left(\widetilde{h}_{i}+\delta_{i}^{*}\right)^{H} .
\end{aligned}
$$

Considering the rank of $Z_{k}$, the following result can be derived $\operatorname{Rank} Z_{k} \geq \operatorname{Rank}\left(I+\sum_{i \neq k} \lambda_{i}\left(\widetilde{h}_{i}+\delta_{i}^{*}\right)\left(\widetilde{h}_{i}+\delta_{i}^{*}\right)^{H}\right)-$ $\operatorname{Rank}\left(\left(1 / \gamma_{k}\right) \lambda_{k}\left(\widetilde{h}_{k}+\delta_{k}^{*}\right)\left(\widetilde{h}_{k}+\delta_{k}^{*}\right)^{H}\right) \geq M-1$. Since $\left\{G_{k}\right\}$ is the solution to (SDR), then $\operatorname{Rank}\left(G_{k}\right) \geq 1$ obviously. According to the complementarity slackness condition $\operatorname{Tr}\left(Z_{k} G_{k}\right)=0$ in the KKT condition, we have $\operatorname{Rank} Z_{k} \leq M-1$. So it follows that $\operatorname{Rank} Z_{k}=M-1$, and then $\operatorname{Rank} G_{k}=1$. This completes the proof.

From Theorem 8, it is easy to see that if (SDR) is strictly feasible, then under Assumption A, the dual problem (DSDR) is equivalent to the following instance problem denoted by $\left(\mathrm{D}-\mathrm{SDR}^{\prime}\right)$ :

$$
\begin{array}{ll}
\max & \sum_{k=1}^{K} \lambda_{k} \sigma_{k}^{2} \\
\text { s.t. } \quad & \lambda_{k} \geq 0, \\
& Z_{k}=I-\frac{1}{\gamma_{k}} \lambda_{k}\left(\widetilde{h}_{k}+\delta_{k}^{*}\right)\left(\widetilde{h}_{k}+\delta_{k}^{*}\right)^{H} \\
& +\sum_{i \neq k} \lambda_{i}\left(\widetilde{h}_{i}+\delta_{i}^{*}\right)\left(\widetilde{h}_{i}+\delta_{i}^{*}\right)^{H} \geq 0, \\
& k=1, \ldots, K .
\end{array}
$$

Also note that the dual of this deterministic problem $\left(\mathrm{D}-\mathrm{SDR}^{\prime}\right)$ has the following form:

$$
\begin{array}{ll}
\min _{\left\{G_{k}\right\}_{k=1}^{K}} & \sum_{k=1}^{K} \operatorname{Tr}\left(G_{k}\right) \\
\text { s.t. } & \frac{\left(\widetilde{h}_{k}+\delta_{k}^{*}\right)^{H} G_{k}\left(\widetilde{h}_{k}+\delta_{k}^{*}\right)}{\sum_{i \neq k}\left(\widetilde{h}_{k}+\delta_{k}^{*}\right)^{H} G_{i}\left(\widetilde{h}_{k}+\delta_{k}^{*}\right)+\sigma_{k}^{2}} \geq \gamma_{k}, \\
& G_{k} \geq 0, \quad k=1, \ldots, K .
\end{array}
$$

It is known in [2] that the deterministic problem ( $\left.\mathrm{SDR}^{\prime}\right)$ does always have at least one optimum solution where all $G_{k}$ have rank one which also shows that $\left(\mathrm{SDR}^{\prime}\right)$ is actually an equivalent reformulation of (D-SDR'). So we can conclude that under proper assumption, (SDR) always yields rankone optimal solution, which implies that an optimal solution of (RP) can always be obtained from an instance problem $\left(\mathrm{SDR}^{\prime}\right)$.

\section{Simulation Results}

In this section, some simulation results are presented to illustrate the correctness of our conclusions and the performance of the proposed SDR method. In the simulations, the base station has four antennas (i.e., $M=4$ ) and serves four users (i.e., $K=4$ ); the channel coefficients are i.i.d complex Gaussian random variables with zero mean and unit variance. The channel uncertainty bounds $\epsilon_{k}$ are all 0.01 ; the noise variances of all users are set equally to be 0.1 . We also assume that the stations have the same target SINRs; that is, $\gamma_{1}=\gamma_{2}=$ $\gamma_{3}=\gamma_{4}=\gamma$.

In the first experiment, we investigate the feasibility rate of the SDR. For each data point of $\gamma$, we generate 500 realizations of the channel estimates $\left\{\widetilde{h}_{i}\right\}_{i=1}^{4}$. Figure 1 displays 


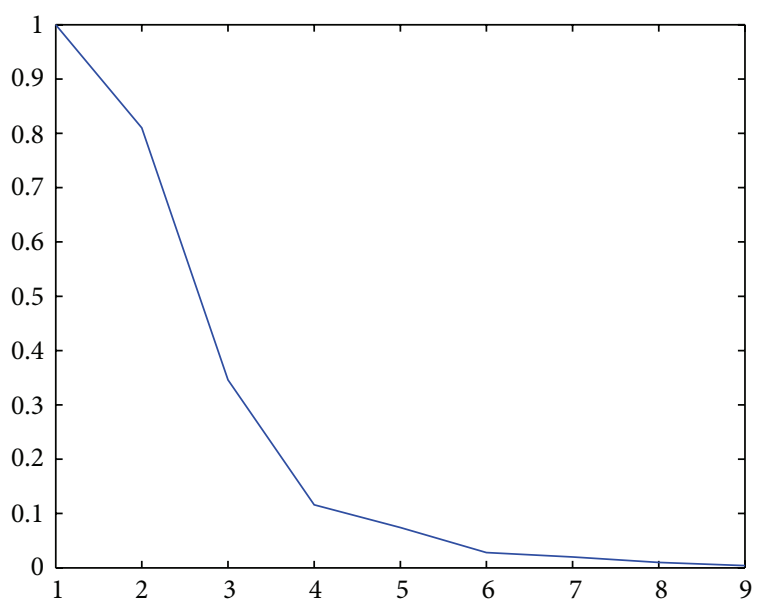

FIGURE 1: Feasibility rate (\%) versus target SINR $\gamma$.

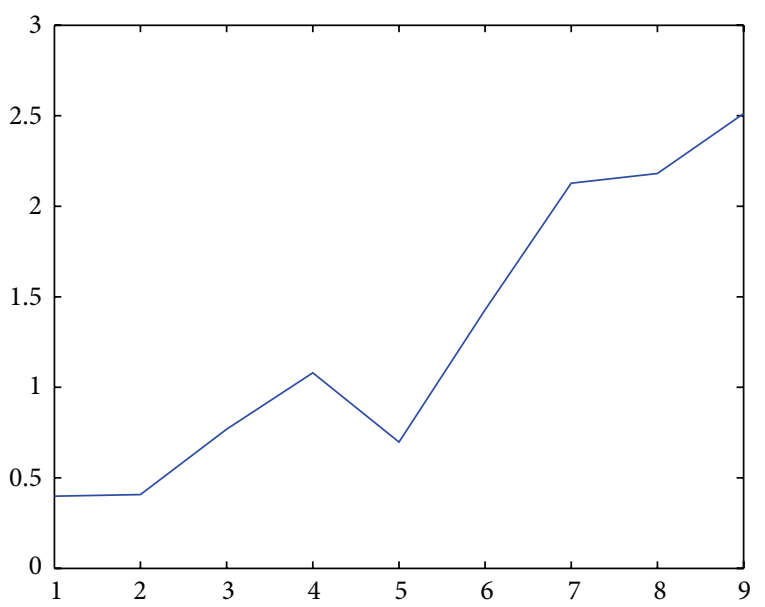

FIGURE 2: Smallest eigenvalue of $X_{k}+\alpha_{k} I$ versus target SINR $\gamma$.

the simulation results. As seen, the feasibility rate of SDR will decrease as the value of $\gamma$ increases. The value of $\gamma$ is chosen as $1,2,3,4,5,6,7,8,9$.

In the second experiment, we examine whether Assumption A (or equivalently B, C) holds in Theorem 8 . As we know, if at the optimal solution $\left\{G_{k}\right\}\left\{\alpha_{k}\right\}$, the matrix $X_{k}+\alpha_{k} I$ is positive definite, then it is obvious that the assumption holds. So we just need to identify whether the solution matrix $X_{k}+$ $\alpha_{k} I$ is positive definite. In our numerical experiment, for each data point of $\gamma$, and the above generated 500 realizations of $\left\{\widetilde{h}_{i}\right\}_{1}^{4}$, we calculate the minimum of all smallest eigenvalues of $X_{k}+\alpha_{k} I$ for $k=1, \ldots, 4$. Figure 2 displays that all the matrices $X_{k}+\alpha_{k} I$ are positive definite in all cases when YALMIP can find the solution of SDR.

To demonstrate the tightness of SDR method, we compute the second eigenvalue defined as $\tau=\max _{k=1,2,3,4} \tau_{2}\left(G_{k}\right)$ where $\tau_{2}\left(G_{k}\right)$ denotes the second largest eigenvalue of $G_{k}$. For each data point of $\gamma$, and the generated 500 realizations, the simulation result is shown in Figure 3. Figure 3 displays that the maximum of all the second largest eigenvalue of $G_{k}$ is very close to zero, which means that the solution of

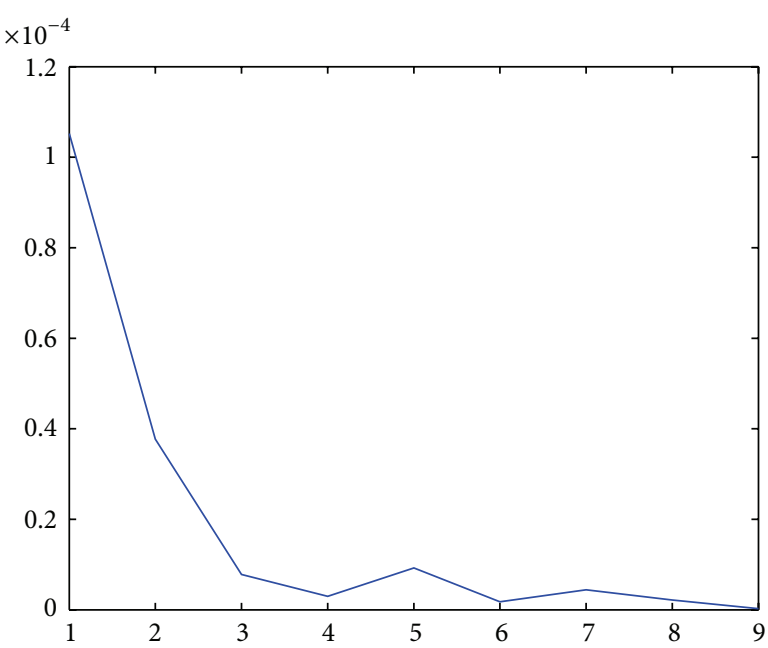

FIgURE 3: Second eigenvalue $\tau$ versus target $\operatorname{SINR} \gamma$.

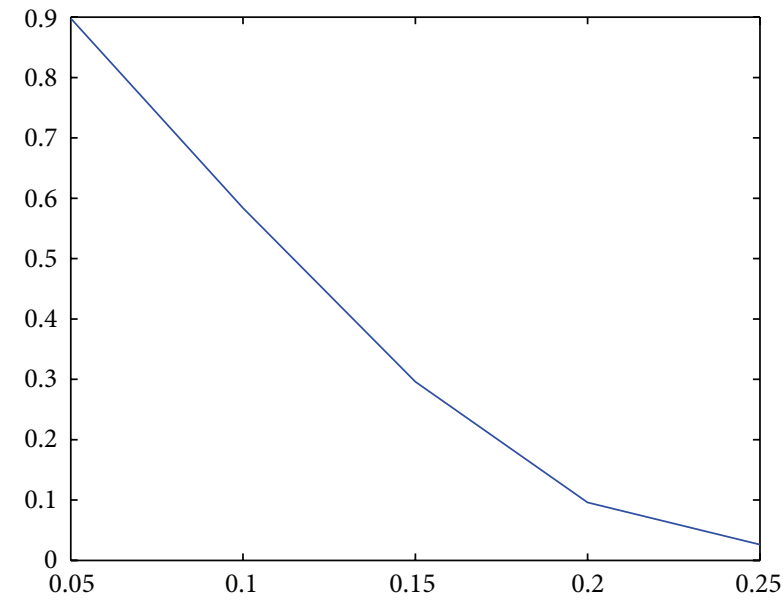

FIGURE 4: Probability of rank-one solution against the error bound $\epsilon_{k}$.

the SDR method is indeed rank one combining the fact in the second experiment that the assumption holds in this simulation. From Figure 3, we see that when $\gamma=1$ or 2, we have $\tau=0.1052 * 10^{-3}$ and $0.377 * 10^{-4}$. From theoretical aspect, the value of $\tau$ should exactly be zero since $X_{k}+\alpha_{k} I$ is positive definite. It is the computation error that result in this phenomenon, especially when the value of $\gamma$ is small.

For fixed $\gamma_{k}=1(k=1,2,3,4)$, we also assume that the stations have the same error bound; that is, $\epsilon_{1}=\epsilon_{2}=\epsilon_{3}=$ $\epsilon_{4}=\epsilon$. It is obvious that probability of rank-one solution against the error bound $\epsilon$ decreases as the $\epsilon$ increases. To show this, we let the value of $\epsilon$ be chosen as $0.05,0.1,0.15,0.2$, and 0.25 . We demonstrate the probability of the rank-one solution in the 500 realizations of the channel estimates against the error bound. In Figure 4, we can see that the probability of the rank-one solution decreases remarkably as the $\epsilon$ increases.

At last, we provide a counterexample whose solutions are not rank one. In this counterexample, the three channel vectors are $\widetilde{h}_{1}=\left[\begin{array}{c}0.7063-1.0078 i \\ -0.9102-0.0127 i \\ -0.5121-0.9088 i\end{array}\right], \widetilde{h}_{2}=\left[\begin{array}{c}0.3312-0.7232 i \\ -0.8027+0.5017 i \\ 0.5206+0.5656 i\end{array}\right]$, and 
$\tilde{h}_{3}=\left[\begin{array}{c}0.1859-0.1013 i \\ 0.4601-0.1435 i \\ 0.2953+0.2650 i\end{array}\right]$ with SINR requirement $\gamma_{1}=\gamma_{2}=\gamma_{3}=1$ and $\sigma_{1}=\sigma_{2}=\sigma_{3}=1, \epsilon_{1}=\epsilon_{2}=\epsilon_{3}=0.3$. The best objective value is 427.109965 . But the eigenvalue of $G_{3}$ is $\left[\begin{array}{lll}0.0001 & 65.7194 & 147.2167\end{array}\right]^{T}$.

The existence of counterexample shows that the guess that the solutions of the (SDR) must be rank one is wrong, except that some assumptions (such as the one proposed in this paper) hold.

In our simulation, we focus on the performance of SDP relaxation method; that is, under proper assumptions, the (SDR) always yields rank-one solution, which also means that the (SDR) can provide globally optimum solutions of the primal robust transmit beamforming problem.

\section{Conclusion}

This paper considers the SDP relaxation to the robust beamforming problem. The main contribution is to get a sufficient condition which can assure the tightness of the SDP relaxation. This condition is that at the optimal solution, there exists a unique error vector $\delta_{k}^{*}$ which satisfies some necessary conditions. From the simulation, it can be seen that in most cases of the 500 realizations of the channel estimates this condition holds when the rank-one solution is achieved. This phenomenon inspires us to get more insight into what is happening here at the optimal solution. We also think it is possible to use these conditions to generate some other conditions that are easier to use in practice. This will be a research direction in the future.

\section{Acknowledgments}

This research was supported by Innovation Program of Shanghai Municipal Education Commission (12ZZ071), Shanghai Pujiang Program (11PJC059), and NSFC (11271243).

\section{References}

[1] A. Goldsmith, S. A. Jafar, N. Jindal, and S. Vishwanath, "Capacity limits of MIMO channels," IEEE Journal on Selected Areas in Communications, vol. 21, no. 5, pp. 684-702, 2003.

[2] M. Bengtsson and B. Ottersten, "Optimal and suboptimal transmit beamforming," in Handbook of Antennas in Wireless Communications, L. C. Godara, Ed., chapter 18, CRC Press, Boca Raton, Fla, USA, 2001.

[3] A. Wiesel, Y. C. Eldar, and S. Shamai, "Linear precoding via conic optimization for fixed MIMO receivers," IEEE Transactions on Signal Processing, vol. 54, no. 1, pp. 161-176, 2006.

[4] A. Gershman, N. Sidiropoulos, S. Shahbazpanahi, M. Bengtsson, and B. Ottersten, "Convex optimization-based beamforming," IEEE Signal Processing Magazine, vol. 27, no. 3, pp. 62-75, 2010.

[5] M. B. Shenouda and T. N. Davidson, "Probabilisticallyconstrained approaches to the design of the multiple antenna downlink," in Proceedings of the 42nd Asilomar Conference on Signals, Systems and Computers, pp. 1120-1124, Pacific Grove, Calif, USA, October 2008.
[6] N. Vučić and H. Boche, "A tractable method for chanceconstrained power control in downlink multiuser MISO systems with channel uncertainty," IEEE Signal Processing Letters, vol. 16, no. 5, pp. 346-349, 2009.

[7] A. Mutapcic, S.-J. Kim, and S. Boyd, "A tractable method for robust downlink beamforming in wireless communications," in Proceedings of the 41st IEEE Asilomar Conference on Signals, Systems and Computers (ACSSC '07), pp. 1224-1228, Pacific Grove, Calif, USA, November 2007.

[8] A. Abdel-Samad, T. N. Davidson, and A. B. Gershman, "Robust transmit eigen beamforming based on imperfect channel state information," IEEE Transactions on Signal Processing, vol. 54, no. 5, pp. 1596-1609, 2006.

[9] M. B. Shenouda and T. N. Davidson, "Nonlinear and linear broadcasting with QoS requirements: tractable approaches for bounded channel uncertainties," IEEE Transactions on Signal Processing, vol. 57, no. 5, pp. 1936-1947, 2009.

[10] W. Uditha, M. Codreanu, and M. Latva-aho, "Robust beamformer design for underlay cognitive radio network using worst case optimization," in Proceedings of the International Symposium on Modeling \& Optimization in Mobile, Ad Hoc \& Wireless Networks (WiOpt '13), pp. 404-411, 2013.

[11] N. Vučić and H. Boche, "Robust QoS-constrained optimization of downlink multiuser MISO systems," IEEE Transactions on Signal Processing, vol. 57, no. 2, pp. 714-725, 2009.

[12] G. Zheng, K. K. Wong, and T. S. Ng, "Robust linear MIMO in the downlink: a worst-case optimization with ellipsoidal uncertainty regions," EURASIP Journal on Advances in Signal Process, vol. 2008, Article ID 609028, pp. 1-15, 2008.

[13] Z.-Q. Luo, W.-K. Ma, A. So, Y. Ye, and S. Zhang, "Semidefinite relaxation of quadratic optimization problems," IEEE Signal Processing Magazine, vol. 27, no. 3, pp. 20-34, 2010.

[14] Z.-Q. Luo and T.-H. Chang, "SDP relaxation of homogeneous quadratic optimization: approximation bounds and applications," in Convex Optimization in Signal Processing and Communications, D. P. Palomar and Y. Eldar, Eds., chapter 4, pp. 117-165, Cambridge University Press, Cambridge, UK, 2010.

[15] C. Shen, T.-H. Chang, K.-Y. Wang, Z. Qiu, and C.-Y. Chi, "Distributed robust multicell coordinated beamforming with imperfect CSI: an ADMM approach," IEEE Transactions on Signal Processing, vol. 60, no. 6, pp. 2988-3003, 2012.

[16] K. Y. Wang, T. H. Chang, W. K. Ma, and C. Y. Chi, "A semidefinite relaxation based conservative approach to robust transmit beamforming with probabilistic SINR constraints," in Proceedings of the European Signal Processing Conference (EUSIPCO '10), pp. 407-411, 2010.

[17] E. Song, Q. Shi, M. Sanjabi, R. Sun, and Z.-Q. Luo, "Robust SINR-constrained MISO downlink beamforming: when is semidefinite programming relaxation tight?" EURASIP Journal on Wireless Communications and Networking, vol. 1, article 243, pp. 1-11, 2012.

[18] T. H. Chang, W. K. Ma, and C. Y. Chi, "Worst-case robust multiuser transmit beamforming using semidefinite relaxation: duality and implications," in Proceedings of the 45th IEEE Asilomar Conference on Signals, Systems and Computers, 2011.

[19] S. Boyd and L. Vandenberghe, Convex Optimization, Cambridge University Press, Cambridge, UK, 2004.

[20] S. Boyd, L. El Ghaoui, E. Feron, and V. Balakrishnan, Linear Matrix Inequalities in System and Control Theory, vol. 15 of SIAM Studies in Applied Mathematics, SIAM, Philadelphia, Pa, USA, 1994. 


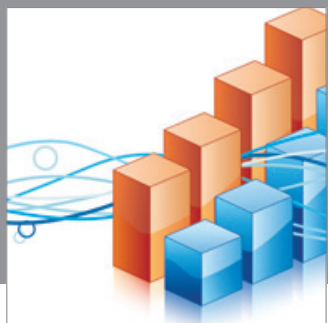

Advances in

Operations Research

mansans

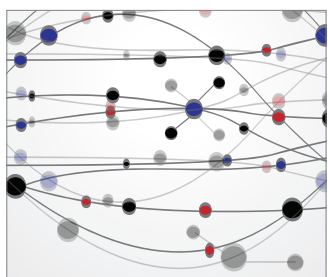

The Scientific World Journal
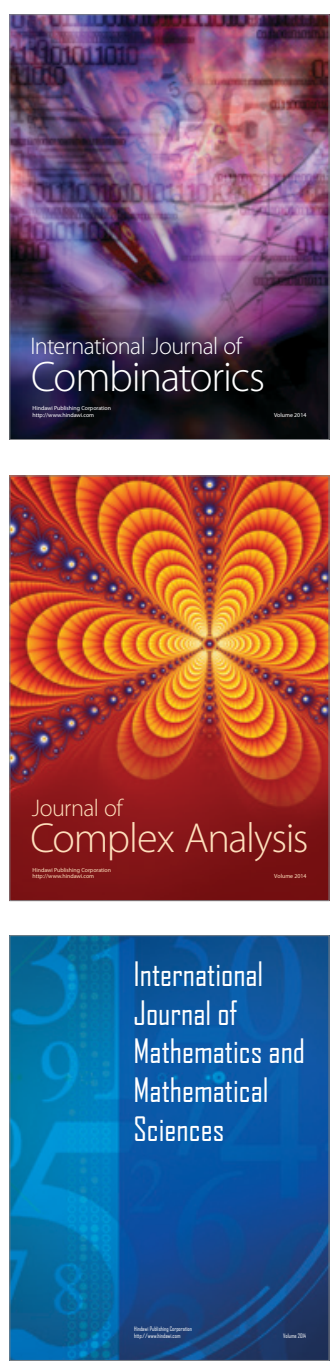
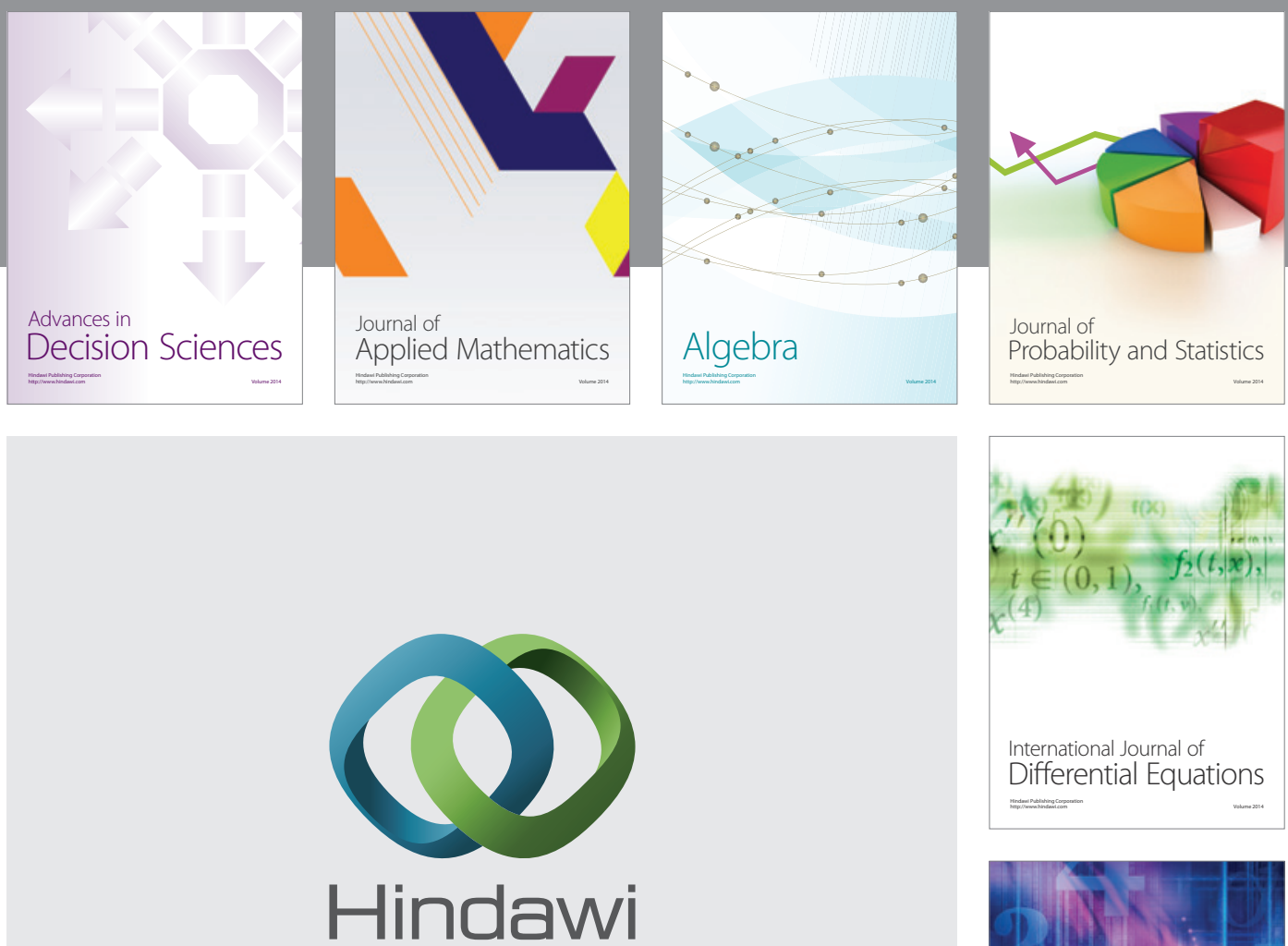

Submit your manuscripts at http://www.hindawi.com
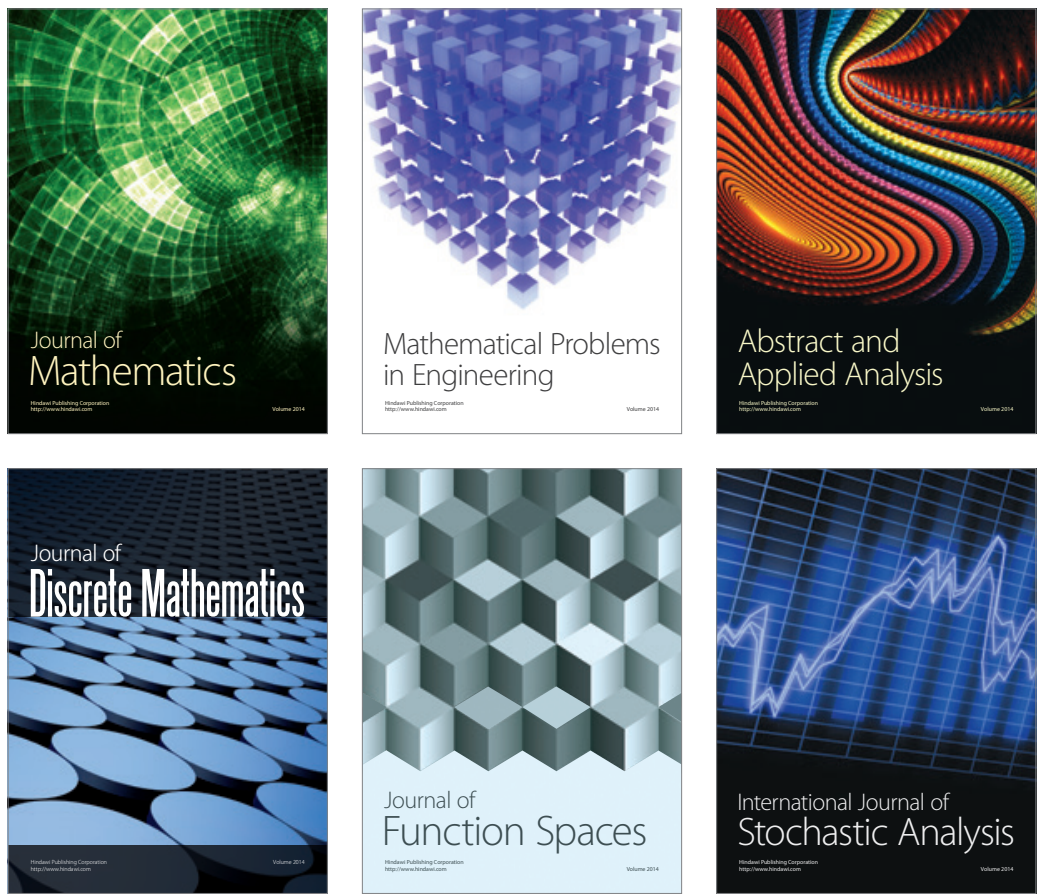

Journal of

Function Spaces

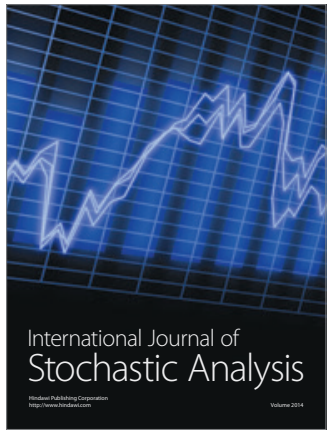

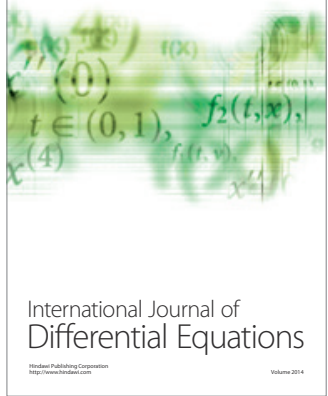
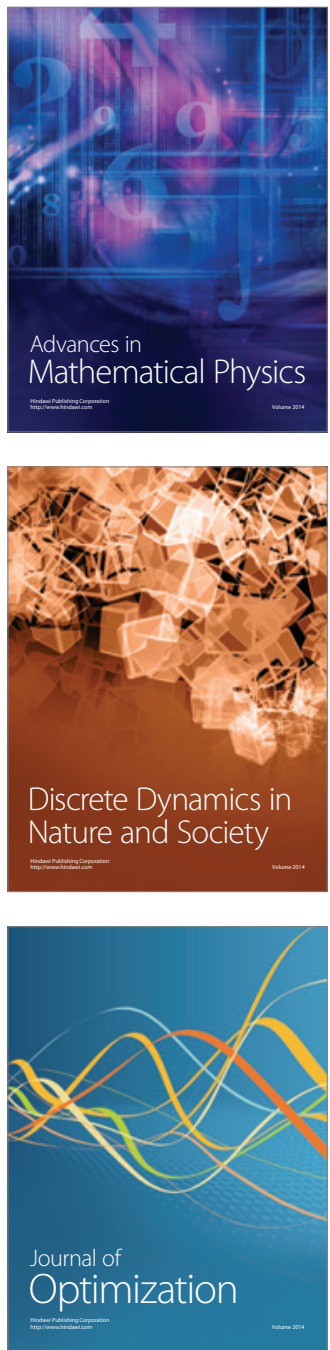